\title{
Development of The Viking Speech Scale to classify the speech of children with cerebral palsy
}

\author{
Lindsay Pennington ${ }^{\mathrm{a}, 1, *}$, Daniel Virella ${ }^{\mathrm{b}, 1}$, Tone Mjøen ${ }^{\mathrm{c}, 1}$, \\ Maria da Graça Andrada ${ }^{\mathrm{d}, 1}$, Janice Murray ${ }^{\mathrm{e}, 1}$, Allan Colver ${ }^{\mathrm{a}, 1}$, \\ Kate Himmelmann f,1, Gija Rackauskaite ${ }^{\mathrm{g}, 1}$, Andra Greitane ${ }^{\mathrm{h}, 1}$, \\ Audrone Prasauskiene ${ }^{\mathrm{i}, 1}$, Guro Andersen ${ }^{c, 1}$, Javier de la Cruz ${ }^{\mathrm{j}, 1}$ \\ ${ }^{a}$ Institute of Health and Society, Newcastle University, Sir James Spence Institute, Royal Victoria Infirmary, \\ Newcastle upon Tyne NE1 4LP, UK \\ ${ }^{\mathrm{b}}$ Hospital de Dona Estefânia, Centro Hospitalar de Lisboa Central, Rua Jacinta Marto, 1169-045 Lisboa, Portugal \\ ${ }^{\mathrm{c}}$ Vestfold Hospital Trust, Postbox 2168, N-3103 Tønsberg, Norway \\ ${ }^{\mathrm{d}}$ Federação das Associações Portuguesas de Paralisia Cerebral, Avenida Rainha Dona Amélia, 1600-676 Lisboa, Portugal \\ ${ }^{\mathrm{e}}$ Faculty of Health, Psychology \& Social Care, Manchester Metropolitan University, Hathersage Road, Manchester M13 0JA, UK \\ ${ }_{\mathrm{f}}^{\mathrm{f}}$ Department of Pediatrics, Institute of Clinical Sciences, Queen Silvia Children's Hospital, Sahlgrenska Academy at the University of \\ Gothenburg, Göteborg, Sweden \\ ${ }^{g}$ Department of Paediatrics, University Hospital, Aarhus, Denmark \\ ${ }^{\mathrm{h}}$ Rehabilitation Center "Mes esam lidzas", Riga 1039, Latvia \\ ${ }^{i}$ Children's Rehabilitation Hospital affiliated to the Hospital of Lithuanian University of Health Sciences, Kaunas, Lithuania \\ ${ }^{j}$ Clinical Research Unit, Imas12-Ciberesp, Hospital 12 Octubre, Madrid, Spain
}

\section{A R T I C L E I N F O}

\section{Article history:}

Received 17 April 2013

Received in revised form 22 June 2013

Accepted 25 June 2013

Available online 24 July 2013

\section{Keywords:}

Cerebral palsy

Surveillance

Speech

Dysarthria

Children

Classification

\begin{abstract}
A B S T R A C T
Surveillance registers monitor the prevalence of cerebral palsy and the severity of resulting impairments across time and place. The motor disorders of cerebral palsy can affect children's speech production and limit their intelligibility. We describe the development of a scale to classify children's speech performance for use in cerebral palsy surveillance registers, and its reliability across raters and across time. Speech and language therapists, other healthcare professionals and parents classified the speech of 139 children with cerebral palsy ( 85 boys, 54 girls; mean age 6.03 years, SD 1.09) from observation and previous knowledge of the children. Another group of health professionals rated children's speech from information in their medical notes. With the exception of parents, raters reclassified children's speech at least four weeks after their initial classification. Raters were asked to rate how easy the scale was to use and how well the scale described the child's speech production using Likert scales. Inter-rater reliability was moderate to substantial ( $k>.58$ for all comparisons). Test-retest reliability was substantial to almost perfect for all groups $(k>.68)$. Over $74 \%$ of raters found the scale easy or very easy to use; $66 \%$ of parents and over $70 \%$ of health care professionals judged the scale to describe children's speech well or very well. We conclude that the Viking Speech Scale is a reliable tool to describe the speech performance of children with cerebral palsy, which can be applied through direct observation of children or through case note review.
\end{abstract}

(c) 2013 Elsevier Ltd. All rights reserved.

\footnotetext{
* Corresponding author. Tel.: +44 1912821360; fax: +44 1912824725.

E-mail addresses: lindsay.pennington@ncl.ac.uk (L. Pennington), danielvirella@oninetspeed.pt (D. Virella), tone.mjoen@siv.no (T. Mjøen), mgcandrada@gmail.com (M. da Graça Andrada),j.murray@mmu.ac.uk (J. Murray), allan.colver@ncl.ac.uk (A. Colver),kate.himmelmann@vgregion.se (K. Himmelmann), gijarack@rm.dk (G. Rackauskaite), andra@edi.lv (A. Greitane), prasauskiene.a@takas.lt (A. Prasauskiene), guro.andersen@siv.no (G. Andersen), jdlcruz@h12o.es (J. de la Cruz).
} 


\section{Introduction}

\subsection{Cerebral palsy}

Cerebral palsy is defined as a permanent but not unchanging disorder of movement and/or posture and of motor function, due to a non-progressive interference/lesion/abnormality of the developing/immature brain (Surveillance of Cerebral Palsy in Europe, 2000). It is the most common cause of motor disorder in childhood affecting around 2-3 per thousand live births (Cans, De-la-Cruz, \& Mermet, 2008). "The motor disorders of cerebral palsy are often accompanied by disturbances of sensation, cognition, communication, perception, and/or behaviour, and/or by a seizure disorder” (Rosenbaum et al., 2007).

\subsection{Surveillance of cerebral palsy}

International surveillance of cerebral palsy monitors trends in the prevalence of cerebral palsy and measures the functional severity of the resulting impairments, in order to inform health and social care policy and practice. Regional registers collect information on factors relating to birth (e.g. gestational age, birth weight), type and distribution of motor disorder, presence and severity of accompanying impairments such as vision and hearing, and performance. For registration purposes confirmation of a diagnosis of cerebral palsy and assessment of children's function usually occurs after age four, to allow for resolution of transient anomalies or diagnosis of slowly progressive disorders and the appearance of clinical features not manifest in the first years of life. The network of European registries - Surveillance of Cerebral Palsy in Europe (SCPE) - agreed that five years was the optimal age for confirmation of diagnosis and case registration (Surveillance of Cerebral Palsy in Europe, 2000).

Surveillance registers vary in their method of data collection. In some registers, clinicians complete questionnaires on children's diagnoses, impairment and function and return this information to the surveillance centre. In other registers, surveillance centre staff (who may not be clinicians) extract information from children's health records (EURO-PERISTAT, 2008). Extensive, clinical assessments are rarely practicable for surveillance purposes because of the time taken for completion and variation in personnel reporting data. Easy to use scales have been developed to describe the gross motor performance (Palisano et al., 1997) and manual performance (Beckung \& Hagberg, 2002; Eliasson et al., 2006) of children with cerebral palsy and are now used across surveillance registers rather than detailed clinical assessments such as the Gross Motor Function Measure (Russell et al., 1993) or the ABILHAND-Kids (Arnould, Penta, Renders, \& Thonnard, 2004). The use of common, consistent measures by registers has enabled the comparison of prevalence rates by severity of impairment across time and regions (Arneson et al., 2009; Platt et al., 2007; SCPE, 2002).

\subsection{Speech, communication and cerebral palsy}

Two systems to classify children's communication have been developed. One describes children's performance in sending and receiving messages (Hidecker et al., 2011); the other rates expression only (Barty \& Caynes, 2009). These scales classify children's success in communicating information using their usual modes of communication. For children with cerebral palsy, communication may be accomplished via multiple modes, such as speech, vocalisation, and aided or unaided augmentative and alternative communication systems. As cerebral palsy always involves a motor disorder and children may also have other developmental difficulties, for surveillance purposes it is important to know the extent to which children's communication difficulties are associated with motor speech disorder.

The motor disorders of cerebral palsy may affect the speed, range, strength, coordination and accuracy of movements of the vocal tract, leading to the motor speech disorder dysarthria (Duffy, 2005). Control of all speech systems - respiration, phonation, resonance, articulation and prosody - may be impaired (Patel, 2003; Yorkston, Beukelman, Strand, \& Bell, 1999). For example, children with cerebral palsy may have shallow, irregular breathing for speech (for instance speaking on small pockets of residual air); their voice may sound harsh and have little pitch variation; air may escape through their nose during speech and they may have a reduced range of vowels and consonants that they can produce clearly. Severity ranges from mild with slight imprecision of speech movements to profound with inability to coordinate the subsystems to produce any recognisable words. The speech systems may be differentially affected, for example respiration may be insufficient to support clear speech but articulation of a range of consonants may be possible. Impairments are usually more severe for children with dyskinetic cerebral palsy than those with spastic forms, but most of the perceptual characteristics (e.g. harshness of voice, mono-pitch) are observed in the speech of children across the different types of cerebral palsy (Love, 2000; Workinger \& Kent, 1991).

Speech production can be measured at the individual speech subsystem level. For example, schemes have been developed to rate impairment of phonation through perceptual vocal profile scales rating harshness and aesthaenia (Hirano, 1981). However, a global measure is necessary for epidemiological surveillance purposes. The global measure should measure speech performance, that is, how speech is produced in daily life to communicate information. Such a scheme should classify

\footnotetext{
${ }^{1}$ On behalf of Surveillance of Cerebral Palsy in Europe Network. See Appendix A.
} 
the perceptual characteristics of speech associated with underlying function of speech subsystems (respiration, phonation, etc.) but might also include speech intelligibility, as the purpose of speech is to convey information (Dykstra, Hakel, \& Adams, 2007; HUI, 2003).

\subsection{Review of speech classification systems}

We undertook a review of the literature to investigate if global scales of speech function in childhood dysarthria had been developed and tested in terms of their reliability and validity. In June 2010 we searched for speech classification tools via Medline, CINAHL, EMBASE, PsychInfo, Web of Knowledge, Scopus, First Search, ERIC, Linguistics and Language Behaviour Abstracts and DARE, searching papers that were indexed under the terms speech production measurement/speech articulation tests or papers that included participants with cerebral palsy and were indexed under the terms speech disorders, articulation disorders, communication disorders or dysarthria. We also hand-searched the following journals from their inception or from 1980 until end March 2010: American Journal of Speech-Language Pathology; Augmentative and Alternative Communication; Developmental Medicine and Child Neurology; Folia Phoniatrica; International Journal of Language and Communication Disorders; International Journal of Rehabilitation Research; Journal of Communication Disorders; Journal of Medical Speech-Language Pathology; Journal of Speech, Language and Hearing Research; Speech, Language and Hearing in Schools. (The current titles are given for journals experiencing name changes since 1980.)

We found two scales. The Speech Production Rating Scale (SPRS) (Pennington \& McConachie, 2001) classifies children's speech according to three criteria: severity of motor speech disorder, phonemic structure of words produced, and intelligibility to familiar and unfamiliar adults in and out of context. Inter-rater reliability between two experienced speech and language therapists was calculated using percentage agreement (83\%), therefore failing to correct for chance agreement. The scale's reliability has not been tested with other groups of raters who may complete surveillance measures and who may be less familiar with linguistic concepts such as word structure. Furthermore, it is unclear how raters should prioritise the different dimensions captured within the scale when classifying children's performance and how impairments of speech subsystems other than articulation, such as respiration and phonation are accommodated. Andersen, Mjøen and Vik classified the speech of children on the Norwegian cerebral palsy register according to their speech clarity using a five point scale (normal speech, slightly indistinct, obviously indistinct, severely indistinct, no verbal speech) (Andersen, Mjøen, \& Vik, 2010). This scale would seem to relate closely to articulation and no other speech characteristics are included. No definitions for the five levels, or differentiation between the levels, were provided nor was information on the scale's validity or reliability. Thus neither of the scales was robust in terms of validity, reproducibility and reliability.

\section{Aim}

The aim of this study was to develop a scale to indicate the presence of a motor speech disorder and speech performance for use in cerebral palsy surveillance. The objectives were to test the face validity of the speech scale, its content validity, its test-retest reliability and its inter-rater reliability. As surveillance registers often collect data using information from case notes, we aimed to compare the agreement between ratings of children's speech from direct observation and from information recorded in case notes. The study is part of SCPE-NET (http://www.scpenetwork.eu), a three-year programme to promote best practice in describing children with cerebral palsy and to document variations in access to health care and in health outcomes. It follows directly from earlier SCPE recommendations for consistent description of children with cerebral palsy (Cans et al., 2007).

\section{Materials and methods}

\subsection{Development of the scale: content validity}

An international expert group comprising two speech and language therapists, one occupational therapist and one neurodevelopmental paediatrician developed the initial scale. It was decided that the original version of the scale would be in English. We took as our starting point the speech of children with cerebral palsy with whom we worked (including live observations, videotaped observations and audio recordings) and descriptions of speech from research papers involving children and adults with cerebral palsy. We wanted the scale to classify the perceptual characteristics of children's speech and the severity of motor speech disorder. We therefore differentiated levels in the classification by the extent to which speech subsystems - respiration, phonation, resonance, articulation and prosody - were affected by speech motor disorder. As speech is used for the purpose of communication we also classified the intelligibility of speech. We considered intelligibility to unfamiliar listeners only, to allow for coding without observation of the child outside clinical environments and to reduce complexity. Preliminary versions of the scale were discussed verbally and via email by the development group. Any disagreements led to changes in wording of the levels. The penultimate iteration of the scale was reviewed by three further content experts (specialist speech and language therapists working with children with cerebral palsy) for face validity. The feedback from these experts led to a change in order of the information in the descriptors, with intelligibility being described first in levels II-IV. 
The finalised scale - The Viking Speech Scale - comprised four levels:

I. Speech is not affected by motor disorder.

II. Speech is imprecise but usually understandable to unfamiliar listeners. Loudness of speech is adequate for one to one conversation. Voice may be breathy or harsh sounding but does not impair intelligibility. Articulation is imprecise; most consonants are produced, but deterioration is noticeable in longer utterances. Although difficulties are noticeable, speech is usually understandable to unfamiliar listeners out of context.

III. Speech is unclear and not usually understandable to unfamiliar listeners out of context. Difficulties controlling breathing for speech - can produce one word per utterance and/or speech is sometimes too loud or too quiet to be understood. Voice may be harsh sounding; pitch may change suddenly. Speech may be markedly hyper nasal. A very small range of consonants are produced. The severity of the difficulties makes the speech difficult to understand out of context.

IV. No understandable speech.

Explanations of the differences between levels I and II and levels II and III were provided in the scale. The full scale can be found at http://www.scpenetwork.eu/en/about-scpe/scpe-net-project/harmonisation/communication/.

The scale was translated into Danish, Latvian, Lithuanian, Norwegian, Portuguese (Portugal), Spanish (Spain) and Swedish, following international guidelines that included two independent translators, discussions on phrasing and terminology by two focus-groups (parents and health professionals) and back translation, to ensure retention of original concepts and meaning (Beaton, Bombardier, Guillemin, \& Ferraz, 1976). Examples of phoneme substitutions given to describe typically developing speech were adapted to each language. Back translations were checked and approved by the first author. Focus groups agreed that the scale reflected speech impairment and its impact on intelligibility in each language, and that levels within the scale were differentiable.

\subsection{Application of the scale - psychometric testing}

To test the content validity and reliability of the scale we asked parents (or caretakers), speech and language therapists and other healthcare professionals to apply the scale to classify the speech of children with cerebral palsy and rate their experience of applying the scheme.

\subsubsection{Participants: children}

The speech of a convenience sample of 139 children aged four to thirteen years ( 85 boys, 54 girls, mean age 6.03 years, SD 1.09 years) with cerebral palsy was rated for the study. The children were purposively sampled from seven SCPE surveillance centres (North of England, Portugal (Lisbon and Oporto), Latvia, Lithuania, Norway, Spain (Madrid), Western Sweden), to provide a sample at each centre that varied in clinical type of cerebral palsy, gross motor function and cognitive skills. Denmark did not participate in the sampling of children.

Data on children's motor, sensory and intellectual function were collected using the classification schemes used by SCPE, to show children's wide ranging severity of impairments. Most children had spastic type cerebral palsy $(n=104 ; 76.1 \%$; unilateral $n=35$, bilateral $n=69) ; 28(19.9 \%)$ had dyskinetic type and $7(4.6 \%)$ had ataxic type cerebral palsy. Following usual practice in SCPE surveillance, IQ was classified by paediatricians from clinical observations and medical notes using a three point scale: 41 (29.54\%) children were classified as having an IQ of less than 50; 25 (18.0\%) had IQ 50-69; 56 (40.3\%) had IQ above 70 and the IQ of 17 (12.25\%) was unknown. Gross motor function was classified using the Gross Motor Function Classification System (GMFCS) (Palisano et al., 1997), upper limb function was classified using the Manual Ability Classification System (MACS) (Eliasson et al., 2006) and the Bimanual Fine Motor Function scale (BFMF) (Beckung \& Hagberg, 2002) (Table 1). Vision was classified using a three point scale: 67 (48.2\%) had no visual impairment; 52 (37.4\%) had impairment but not severe; 16 (11.5\%) had severe impairment and the visual function of four (2.9\%) children was unknown. Hearing was classified in the same manner: $122(87.8 \%)$ had no impairment; six (4.3\%) had impairment but not severe; three (2.2\%) had severe impairment and the hearing of eight (5.8\%) children was unknown. Usual modes of communication were recorded: 96 of the children (69.1\%) had some functional speech; 56 (40.3\%) used vocalisations; 34 (24.5\%) used gestures; 22

Table 1

Children's motor function as classified using the GMFCS, MACS and BFMF.

\begin{tabular}{lcll}
\hline Level & GMFCS & BFMF & MACS \\
& $n=139(\%)$ & $n=138(\%)$ & $18(12.9)$ \\
\hline I & $32(23.0)$ & $22(15.8)$ & $33(23.7)$ \\
II & $16(11.5)$ & $25(18.0)$ & $25(18.0)$ \\
III & $27(19.4)$ & $27(19.4)$ & $20(14.4)$ \\
IV & $25(18.0)$ & $20(14.4)$ & $23(16.5)$ \\
V & $34(24.5)$ & $22(15.8)$ & $19(13.7)$ \\
Unknown & $5(3.6)$ & $22(15.8)$ & \\
\hline
\end{tabular}

GMFCS, Gross Motor Function Classification System; BFMF, Bimanual Fine Motor Function Scale; MACS, Manual Ability Classification System. 
(15.8\%) used manual signs; 31 (22.3\%) had a light tech augmentative and alternative communication (AAC) book and 17 (12.2\%) used a high tech AAC device.

\subsubsection{Participants: raters of children's speech}

We aimed for each child's speech to be rated by a parent/caretaker through their knowledge of the child; by a speech and language therapist through direct observation of the child; by one other healthcare professional through direct observation and by a healthcare professional through access to case notes. Speech and language therapists are rare in Norway, and children receive services to address language and communication needs from special educators. The speech of Norwegian children in the study was rated by special educators rather than speech and language therapists. For ease we have referred to the special educators providing language and communication services to children in this study as speech and language therapists. In total 122 children were rated by their caretakers (98 (80.3\%) mothers; 16 (13.1\%) fathers; 2 (1.6\%) other relative; 6 (4.9\%) other caretaker). The speech of 129 children was rated by speech and language therapists (special educators, Norway); 131 were rated by other healthcare professionals from direct observation (22 (16.8\%) physiotherapists; 58 (44.3\%) paediatricians, 51 (38.9\%) other healthcare professionals) and 134 were rated using case notes $(1$ (.7\%) physiotherapist; $1(.7 \%)$ nurse; $112(83.6 \%)$ paediatricians, $20(14.9 \%)$ other health professionals). Of those rating children using case notes, $30(22.4 \%)$ recorded that they had previous knowledge of the child.

\subsubsection{Procedure}

Ethics permission was obtained from each of the participating centres. Families were recruited from clinical caseloads by local clinicians. Parents gave written consent to participate and for their child's skills to be rated for the purpose of the study. Each rater was provided with a copy of the scale in their spoken language. No training on the scale was provided. Each rater classified children's speech skills using the Viking Speech Scale blind to other raters' assessments. At least four weeks later all health professionals were asked to rate each child again, blind to their original rating. Parents completed the scale only once.

To investigate the content validity of the scale each rater was asked to complete Likert ratings of how well the scale describes the performance of producing speech ( 1 = very well; 5 = very badly). The comparison of application of the scale by different groups of raters and through direct observation of the child versus access to case notes enabled us to assess the suitability of the scale for adoption by surveillance registers which collect information in different ways. To enhance this assessment each respondent was asked "How easy did you find the application of this scale in this particular child?" and to answer using a 5 point scale ( 1 = very easy; 2 =easy; 3 = some doubts; $4=$ difficult; $5=$ very difficult).

\subsubsection{Analysis}

Test-retest reliability and inter-rater reliability between rater groups (parent-speech and language therapist; parentother health professionals; speech and language therapists-other health professionals) was assessed using weighted Kappa $(k)$, with $95 \%$ confidence intervals $(95 \% \mathrm{CI})$. Ratings of ease of use and content validity of the scale were compared across raters using Chi square. Analysis was carried out using Stata, Release 11 (StataCorp, 2009).

\section{Results}

\subsection{Agreement of ratings on The Viking Speech Scale between rater groups}

Inter-rater reliability of the Viking Scale was observed to be moderate-substantial for all pairs of rater groups, with Kappa coefficients being in the range of .58-.81, and lower confidence limits being .43 or above, for all pairs of rater groups (Landis \& Koch, 1977) (Table 2 shows kappa results with 95\%CI. Percentage agreement and raw data for the individual levels by pairs of rater groups are provided in the Supplementary data file).

Table 2

Inter-rater reliability using weighted Kappa coefficients with 95\% confidence intervals.

\begin{tabular}{lll}
\hline Rater groups & $n$ & 95\%CI \\
\hline Parents $\times$ SLTs & 126 & .81 \\
Parents $\times$ HCP direct observation & 107 & $.66-.96$ \\
Parents $\times$ HCPs using notes & 117 & $.43-.73$ \\
SLTs $\times$ HCP direct observation & 116 & .74 \\
SLTs $\times$ HCP using notes & 126 & .58 \\
HCP direct observation $\times$ HPC using notes & 123 & .89 \\
\hline
\end{tabular}

SLT, speech and language therapist; HCP, healthcare professional. 


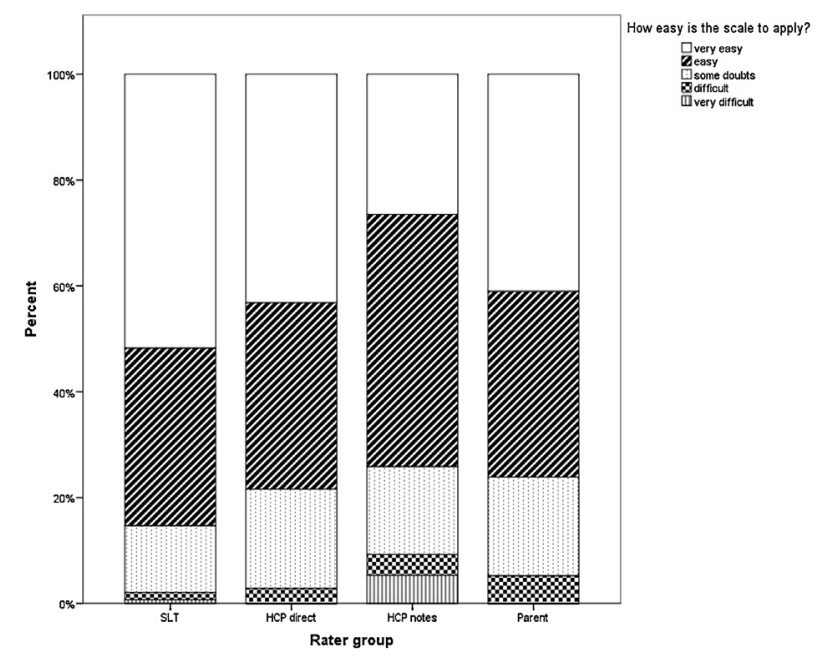

Fig. 1. Ease of application of the Viking Speech Scale by rater group. SLT, speech and language therapist; HCP direct, healthcare professional rating speech from direct observation of the child; HCP notes, healthcare professional rating speech from case notes.

\subsection{Test-retest reliability of The Viking Speech Scale}

Most of the health care professionals who rated the children's speech completed a second rating, blind to their original rating, two to four weeks later. Test-retest reliability was almost perfect for speech and language therapists $(n=97, k=89$, $95 \% \mathrm{CI}=.73-1.0)$, substantial for healthcare professionals by direct observation $(n=72, k=68,95 \% \mathrm{CI}=.50-.87)$ and almost perfect for healthcare professionals using case notes $(n=61, k=92,95 \% \mathrm{CI}=.72-1.00)$ (Landis \& Koch, 1977) (see Supplementary data file for actual agreement).

\subsection{Ease of use of The Viking Speech Scale}

Most raters found the scale easy to apply, with proportionally more speech and language therapists finding the scale easy to use: $74.6 \%$ of parents; $84.5 \%$ of speech and language therapists; $77.6 \%$ of healthcare professionals rating from direct observation and $74.1 \%$ of professionals rating using case notes rated the scale as very easy or easy to apply (Fig. 1). The effect of rater group was statistically significant $\left(\chi^{2}(\mathrm{df} 9)=28.50, p=.001\right)$. A post hoc comparison, combining some doubt/ difficult/very difficult categories, suggested that health professionals rating speech from case notes found the scale most difficult to apply $\left(\chi^{2}(\mathrm{df} 3)=9.78, p=.02\right)$. However, the percentage judging the scale as difficult or very difficult to use was small (9.3\%).

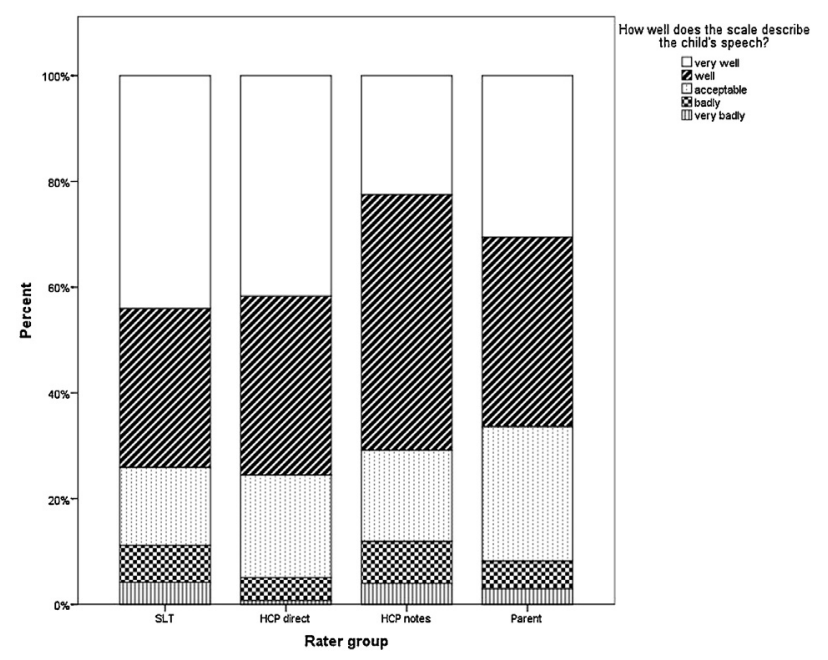

Fig. 2. Raters' perceptions of the fit of the descriptions in the Viking Speech Scale with the child's speech. SLT, speech and language therapist; HCP direct, healthcare professional rating speech from direct observation of the child; HCP notes, healthcare professional rating speech from case notes. 


\subsection{Content validity of The Viking Speech Scale}

Most raters judged the scale to rate speech performance well: $66.4 \%$ of parents, $74.1 \%$ of speech and language therapists, $77.3 \%$ of healthcare professionals rating through direct observation of the child and $70.9 \%$ of healthcare professionals rating children through access to case notes judged the scale to describe the child's speech well or very well (Fig. 2). Again, the effect of rater group was statistically significant $\left(\chi^{2}(\mathrm{df} 9)=29.36, p=.001\right)$. We undertook two post hoc comparisons: $(1)$ compared the combined categories 'very well/well' with all other categories combined. (2) compared the combined categories 'very badly/badly' with all other rating categories combined. Neither comparison showed significant differences between rater groups in judgments on whether the scale described the children's speech well or badly.

\section{Discussion}

This study aimed to develop an easy to use, valid and reliable scale of the speech of children with cerebral palsy for use in the surveillance of cerebral palsy. The scale incorporates the presence of a motor speech disorder and the severity of limitations in speech performance in everyday life. The process of developing the scale was similar to that of other classification scales (Eliasson et al., 2006) and took as its starting point the description of the speech of children with cerebral palsy with whom we worked who had different types of motor disorders and severities of dysarthria. Multidisciplinary experts (parents, speech and language therapists, occupational therapists, physiotherapists and paediatricians) were involved in developing the scale, and agreed on the description of speech at each level and the differentiation between levels, thereby ensuring face validity. Content validity was demonstrated by the high percentage of raters from all groups judging the scale to describe well or very well the speech of the children they were rating.

Inter-rater reliability of the scale between groups from different backgrounds and using different sources of information (knowledge of the child, observation and case notes) was moderate to substantial for all rater pairs (Landis \& Koch, 1977). Most raters found the scale easy to apply and ratings were stable over time. In both inter-rater reliability and test-retest reliability assessments lowest reliability was achieved for healthcare professionals who rated children's speech from observation. This may be due to variability within the healthcare professionals on their knowledge of the children they were rating. In order to evaluate classification of children's abilities in usual surveillance conditions no stipulations were made on whether the health care professionals should have prior knowledge of the children in this study. Lower reliability for this group may also arise from variability in their knowledge of speech development; speech and language therapists who have greatest expertise in speech development, found the scale easiest to use. Further research is needed to test the effect of familiarity with the child being coded, familiarity with the scale, and professional group.

Together these results suggest that the scale is suitable for adoption by cerebral palsy surveillance centres, but that it is best applied by healthcare professionals using children's notes and by parents or speech and language therapists from observation.

The Viking Speech Scale was developed to classify the presence of dysarthria and limitations in speech performance. The scale may complement other scales (Barty \& Caynes, 2009; Hidecker et al., 2011) of communication, showing the extent to which motor speech disorders impact on communication performance.

Like other schemes designed to classify children's performance, such as the GMFCS (Palisano et al., 1997), it relies on information from the domains of both body function and activity within the International Classification of Functioning, Disability and Health (ICF) (WHO, 2001). The scale describes the children's speech performance at the level of the individual speech functions affected by dysarthria: breath support for speech, phonation, articulation and rhythm/prosody (Kim, Martin, Hasegawa-Johnson, \& Perlman, 2010; Love, 2000; Patel, 2002a, 2002b; Solomon \& Charron, 1998). As the purpose of speech is to convey information, levels within the scale are also differentiated according to the extent to which the speech signal can be understood by unfamiliar people - its intelligibility. Intelligibility of speech is coded as an activity ('Producing Communication - Speaking') in the ICF (Dykstra et al., 2007). Children whose speech is delayed in development, but who do not have dysarthria would be classified as 'Level I - speech not affected by motor disorder', even though their intelligibility may be compromised. The inclusion of intelligibility descriptions may make the scale easier to apply by individuals with little theoretical knowledge of speech disorders. However, from the current study it is not clear whether raters are using speech function or speech intelligibility descriptions to assign a level; such understanding would require cognitive interviewing of raters applying the scale (Willis, 1994).

The Viking Speech Scale was designed for and tested with children aged four years and above. This was to ensure that all SCPE surveillance centres, which have a minimum reporting age of four years, could adopt the scale if it were shown to be valid and reliable. The scale contains only one set of levels; no age bands have been created. However, it is acknowledged that at four years children's phonological system, and potentially their intelligibility, will still be developing (Dodd, Holm, Hua, \& Crosbie, 2003) and in the introduction to the scale examples of developmental speech substitutions are given. The scale may be easier to apply with older children, whose speech has matured. However, further research is necessary to examine if age affects reliability and ease of application, and if levels on the Viking are stable over time.

Unlike other classification systems (Beckung \& Hagberg, 2002; Eliasson et al., 2006; Hidecker et al., 2011; Palisano et al., 1997), the Viking Speech Scale contains four levels. The scale's levels were determined by the need to create meaningful categories for epidemiological surveillance. The scale is not intended to be a clinical assessment, but may be helpful when summarising children's speech performance in clinical reports and research. Further research is needed to determine the 
criterion validity of the scale and research is currently underway to assess the association between the Viking Speech Scale and objective measures of children's speech intelligibility (Pennington \& Hustad, in preparation).

A limitation of the study is the low numbers of children in the sample from some of the participating countries. Each centre aimed to recruit 30 children to the study, but for some centres this was not possible for reasons relating either to the size of the area covered or to financial constraints. Although the scale has acceptable overall reliability, it is possible that reliability of the scale differs between countries and languages, in spite of cultural adaptation processes undertaken during translation. Further research would be necessary to test similarity in reliability between countries and languages as the sample in the current study is too small to investigate this issue. On the other hand, its multinational design has the advantages of highlighting the validity of the scale as a tool to be used for international surveillance of cerebral palsy and providing validated versions of the scale in eight different languages.

\section{Conclusion}

From its application with a sample of children with cerebral palsy who had a wide ranging motor, cognitive and sensory skills by healthcare professionals from observation and using notes and by parents from recollection of children's speech, we have shown that the scale has face and content validity, is easy to use and reliable. We conclude that the scale can be applied by cerebral palsy surveillance registers that collect information from clinicians' observations and those that collect information from children's medical notes to describe the speech performance of children with cerebral palsy aged four years and above.

\section{Acknowledgement}

This study was funded by the European Union Health Programme - Grant EAHC 20081307 - 'Surveillance of cerebral palsy in Europe: best practice in monitoring, understanding of inequality and dissemination of knowledge'.

\section{Appendix A}

The following SCPE centres collected data for this study: Denmark (led by Gija Rackauskaite), Latvia (led by Andra Greitane), Lithuania (led by Audrone Prasauskiene), Norway (led by Guro Andersen), Portugal (Lisbon and Oporto, led by Daniel Virella), Spain (Madrid, led by Javier de la Cruz), Sweden (Western Sweden, led by Kate Himmelmann), UK (North of England, led by Karen Horridge).

\section{Appendix B. Supplementary data}

Supplementary data associated with this article can be found, in the online version, at http://dx.doi.org/10.1016/ j.ridd.2013.06.035.

\section{References}

Andersen, G., Mjøen, T. R., \& Vik, T. (2010). Prevalence of speech problems and the use of augmentative and alternative communication in children with cerebral palsy: A registry-based study in Norway. Perspectives on Augmentative and Alternative Communication, 19, 12-20.

Arneson, C. L., Durkin, M. S., Benedict, R. E., Kirby, R. S., Yeargin-Allsopp, M., Van Naarden Braun, K., et al. (2009). Prevalence of cerebral palsy: Autism and Developmental Disabilities Monitoring Network, three sites, United States, 2004. Disability and Health Journal, 2, 45-48.

Arnould, C., Penta, M., Renders, A., \& Thonnard, J. (2004). ABILHAND-Kids: A measure of manual ability in children with cerebral palsy. Neurology, 63, 1045-1052.

Barty, E., \& Caynes, K. (2009). Development of the functional communication classification system. Paper presented at the third international cerebral palsy conference February.

Beaton, B. E., Bombardier, C., Guillemin, F., \& Ferraz, M. B. (1976). Guidelines for the process of cross-cultural adaptation of self-report measures. Spine, 25, 31863191.

Beckung, E., \& Hagberg, G. (2002). Neuroimpairments, activity limitations, and participation restrictions in children with cerebral palsy. Developmental Medicine and Child Neurology, 44, 309-316.

Cans, C., De-la-Cruz, J., \& Mermet, M. (2008). Epidemiology of cerebral palsy. Paediatrics and Child Health, 18, 393-398

Cans, C., Dolk, H., Platt, M. J., Colver, A., Prasauskiene, A., \& Krageloh-Mann, I. (2007). Recommendations from the SCPE collaborative group for defining and classifying cerebral palsy. Developmental Medicine and Child Neurology, 49, 35-38.

Dodd, B., Holm, A., Hua, Z., \& Crosbie, S. (2003). Phonological development: A normative study of British English-speaking children. Clinical Linguistics and Phonetics, 17, 617-643.

Duffy, J. R. (2005). Motor speech disorders: Substrates, differential diagnosis, and management (2nd ed.). Philadelphia, PA: Elsevier Mosby.

Dykstra, A. D., Hakel, M. E., \& Adams, S. G. (2007). Application of the ICF in reduced speech intelligibility in dysarthria. Seminars in Speech and Language, 28, $301-311$

Eliasson, A., Krumlinde Sundholm, L., Rösblad, B., Beckung, E., Arner, M., Öhrvall, A. M., et al. (2006). The Manual Ability Classification System (MACS) for children with cerebral palsy: Scale development and evidence of validity and reliability. Developmental Medicine and Child Neurology, 48, 549-554.

EURO-PERISTAT. (2008). European perinatal health report. <http://www.europeristat.com>.

Hidecker, M. J. C., Paneth, N., Rosenbaum, P. L., Kent, R. D., Lillie, J., Eulenberg, J. B., et al. (2011). Developing and validating the Communication Function Classification System for individuals with cerebral palsy. Developmental Medicine and Child Neurology, 53, 704-710.

Hirano, M. (1981). Clinical examination of voice. Vienna: Springer-Verlag.

HUI, I. (2003). Health Utilities Index Mark 3 (HUI3). Retrieved 25.02.13. 
Kim, H., Martin, K., Hasegawa-Johnson, M., \& Perlman, A. (2010). Frequency of consonant articulation errors in dysarthric speech. Clinical Linguistics and Phonetics, 24, 759-770.

Landis, J., \& Koch, G. (1977). The measurement of observer agreement for categorical data. Biometrics, 33, 159-174.

Love, R. J. (2000). Childhood motor speech disability (2nd ed.). Boston: Allyn \& Bacon.

Palisano, R. J., Rosenbaum, P., Walter, S., Russell, D., Wood, E., \& Galuppi, B. (1997). Development and reliability of a system to classify gross motor function in children with cerebral palsy. Developmental Medicine and Child Neurology, 39, 214-223.

Patel, R. (2002a). Phonatory control in adults with cerebral palsy and severe dysarthria. Augmentative and Alternative Communication, 18, 2-10.

Patel, R. (2002b). Prosodic control in severe dysarthria: Preserved ability to mark the question-statement contrast. Journal of Speech, Language and Hearing Research, 45, 858-870.

Patel, R. (2003). Acoustic characteristics of the question-statement contrast in severe dysarthria due to cerebral palsy. Journal of Speech Language and Hearing Research, 46, 1401-1415

Pennington, L., \& McConachie, H. (2001). Predicting patterns of interaction between children with cerebral palsy and their mothers. Developmental Medicine and Child Neurology, 43, 83-90.

Platt, M. J., Cans, C., Johnson, A., Surman, G., Topp, M., Torrioli, M. G., et al. (2007). Trends in cerebral palsy among infants of very low birthweight ( $<1500 \mathrm{~g})$ or born prematurely ( $<32$ weeks) in 16 European centres: A database study. Lancet, 369, 43-50.

Rosenbaum, P., Paneth, N., Leviton, A., Goldstein, M., Bax, M., Damiano, D., et al. (2007). A report: The definition and classification of cerebral palsy April 2006. Developmental Medicine and Child Neurology, 49, 8-14.

Russell, D., Rosenbaum, P., Gowland, C., Hardy, S., Lane, M., Plews, N., et al. (1993). Gross motor function measure manual (2nd ed.). Kingston, Ontario: School of Rehabilitation, Queens University.

SCPE. (2002). Prevalence and characteristics of children with cerebral palsy in Europe. Developmental Medicine and Child Neurology, 44, 633-640.

Solomon, N. P., \& Charron, S. (1998). Speech breathing in able-bodied children and children with cerebral palsy: A review of the literature and implications for clinical intervention. American Journal of Speech-Language Pathology, 7, 61-78.

StataCorp. (2009). Stata (version 11). College Station, TX: StataCorp.

Surveillance of Cerebral Palsy in Europe. (2000). Surveillance of cerebral palsy in Europe: A collaboration of cerebral palsy surveys and registers. Surveillance of Cerebral Palsy in Europe (SCPE). Developmental Medicine and Child Neurology, 42, 816-824.

WHO. (2001). International classification of functioning, disability and health: ICF. Geneva: WHO.

Willis, G. (1994). Cognitive interviewing and questionnaire design: A training manual. Working Paper \#7. Atlanta, GA: National Center for Health Statistics.

Workinger, M. S., \& Kent, R. D. (1991). Perceptual analysis of the dysarthrias in children with athetoid and spastic cerebral palsy. In C. A. Moore, K. M. Yorkston, \& D. R. Beukelman (Eds.), Dysarthria and apraxia of speech: Perspectives on management (pp. 109-126). Baltimore: Paul Brookes.

Yorkston, K. M., Beukelman, D. R., Strand, E. A., \& Bell, K. R. (1999). Management of motor speech disorders in chidlren and adults. Austin: Pro-ed. 